\title{
Ensuring editorial continuity and quality of science during the COVID-19 storm: the ICM experience
}

\author{
Thomas Bein ${ }^{{ }^{*}}$ and Alessia Vargiolu ${ }^{2,3}$ on behalf of the ICM Editorial Board
}

(0) 2020 The Author(s), corrected publication 2021

\section{Dear Editor,}

High-quality peer reviews are absolutely necessary in medical journals, for publishing quality manuscripts [1]. To guarantee this in view of the increasing volume of scientific production, journals are managed by competent scientific editors who rely on a large number of qualified reviewers. Time, energy, experience, critical thinking and knowledge of the current scientific trends are needed to write a good review [2]. Reviewers are usually unpaid and their efforts are little acknowledged. To compensate this situation, Intensive Care Medicine (ICM) has been rewarding its reviewers for years with EU-CME credits. Identically, many journals are facing difficulties in finding appropriate reviewers who would do the job in a timely manner. The results of the survey, Why do reviewers decline to review? [3], showed that the most important factor responsible for the reviewers' decision not to take up the job was existing workload. Since the outbreak of the SARS-CoV-2-related disease (COVID-19) swept over the intensive care units (ICUs) worldwide in the early months of 2020, we were interested to know whether the massive workload caused by the overwhelming ICU admissions and the confrontation with a new and challenging disease might have limited the peer-review activity of those experts caught in the clinical storm. Therefore, we analyzed the manuscript submissions to ICM and the responses of the invited reviewers (acceptance vs. 'impracticalities', defined as the sum of declines, un-invitations or task terminations due to non-response) from January to April 2020, and compared the findings

\footnotetext{
*Correspondence: thomas.bein@klinik.uni-regensburg.de

${ }^{1}$ Faculty of Medicine, University of Regensburg, Regensburg, Germany

Full author information is available at the end of the article
}

of peer-review activity with the same time span in 2019 (Table 1). From January 1st to April 30th 2020, there was a considerable increase in submissions (1201 total submissions, 617 of which were COVID-related) over the comparable time in 2019 ( 554 total submissions). In both cases, the average percentage of advanced rejections was around 60 . This workload has been managed in-house, coordinated by the Editor-in-Chief on a daily basis. In 2019, 180 manuscripts were sent to 1.271 reviewers. In the comparable period of 2020, 296 manuscripts were sent out to 1.741 reviewers. In 2020, the percentage of impracticalities (declines, un-invitations, or terminations due to non-response, mostly due to work overload) of reviewers on absolute submissions was higher between January and March than in 2019 but it returned to the level of the previous year at the beginning of April (see Electronic Supplemental Fig. 1). Furthermore, there was no difference in the percentage of late reviews (2020: $13.8 \%$; 2019: $15.8 \%, p=0.29)$ or the average number of late days (2020: 2.1 days; 2019: 1.5 days, $p=0.93$ ).

Despite the rapid and massive increase in workload for intensive care health professionals due to the "Corona crisis' at the beginning of 2020, looking "Death in the Eye" [4], our findings suggest that, overall, the peer-review activity in high-quality intensive care journals has not suffered a crisis and does guarantee the continuity of one of the columns of quality in science. Our editorial responsibility is to 'avoid research waste during the COVID-19 pandemic and plan for the future' [5]. It is not foreseeable at the moment, whether and how such an exceptional situation for intensive care medicine will continue, disappear, or even return. We should be grateful to all the committed reviewers of many medical journals: they help to promote science-not only about COVID-19. 
Table 1 Summary of ICM and reviewers workload during January 1st-April 30th 2019 compared to the same period in 2020

\begin{tabular}{|c|c|c|}
\hline & 2019 & 2020 \\
\hline Total submissions & 554 & 1201 \\
\hline Need approval & 0 & $1^{\mathrm{a}}$ \\
\hline Sent back to authors & 0 & $3^{\mathrm{a}}$ \\
\hline Withdrawn & 3 & 2 \\
\hline Managed submissions & 551 & 1195 \\
\hline Accepted in-house (\%) & $16(2.9)$ & $37(3.1)$ \\
\hline Rejected in-house (\%) & $321(58.2)$ & 755 (63.2) \\
\hline Any disposition term without review ${ }^{b}$ & $34(6.2)$ & $107(8.9)$ \\
\hline Sent out for revision & $180(32.7)$ & $296(24.8)$ \\
\hline Total reviewers invited & 1271 & 1741 \\
\hline Accepted invitation (\%) & $853(67.1)$ & $1118(64.2)$ \\
\hline Declined invitation (\%) & $264(20.8)$ & $359(20.6)$ \\
\hline Un-invited before agreeing (\%) & $143(11.2)$ & $210(12.1)$ \\
\hline Terminated before agreeing (\%) & $11(0.9)$ & $54(3.1)$ \\
\hline Average days to response & 0.6 & 0.5 \\
\hline Average days from agreement to review completion & 5 & 4.7 \\
\hline Revisions submitted on time (\%) & $554(64.9)$ & $716(64.1)$ \\
\hline Revisions submitted late (\%) & $206(24.2)$ & $223(19.9)$ \\
\hline Un-invited after agreeing (\%) & $14(1.6)$ & $19(1.7)$ \\
\hline Terminated after agreeing (\%) & $79(9.3)$ & $143(12.8)$ \\
\hline Pending revisions (\%) & $0(0)$ & $17(1.5)^{c}$ \\
\hline
\end{tabular}

Even if the workload is more than doubled in 2020 with respect to the same period in 2019 , the overall capability and efforts made by both ICM Editors and Reviewers dampened the impact of COVID-19 outbreak on the managing process of manuscripts. Rows highlighted with light blue background color show the "impracticalities" reported by reviewers

a At the moment of the present data analysis, there are still some pending submissions in 2020

b It includes the following dispositions given directly by Editors: Change for a letter, Reject and Transfer to ICMx, Revise before review

c At the moment of the present data analysis, there are still some pending revisions in 2020

\section{Electronic supplementary material}

The online version of this article (https://doi.org/10.1007/s00134-020-06207-y) contains supplementary material, which is available to authorized users.

\section{Author details}

${ }^{1}$ Faculty of Medicine, University of Regensburg, Regensburg, Germany. ${ }^{2}$ School of Medicine, University of Milano - Bicocca, Milan, Italy. ${ }^{3}$ Intensive Care Medicine, Editorial Office, Monza, Italy.

\section{Acknowledgements}

ICM Editorial Board: Giuseppe Citerio, Department of Medicine and Surgery, School of Medicine and Surgery, University of Milano - Bicocca, Milan, Italy. Samir Jaber, Department of Anaesthesia and Intensive Care, Montpellier Saint Eloi University Hospital, and PhyMedExp, University of Montpellier, INSERM, CNRS, Montpellier, France. Miet Schetz, Division of Cellular and Molecular Medicine, Clinical Department and Laboratory of Intensive Care Medicine, KU Leuven University, Leuven, Belgium. Anders Aneman, South Western Sydney Clinical School, Faculty of Medicine University of New South Wales, Sydney, Australia. Yaseen Arabi, Intensive Care Department, King Saud bin Abdulaziz University for Health Sciences and King Abdullah International Medical Research Center, Riyadh, Saudi Arabia. Katherine Brown, Cardiac and Critical Care Division, Great Ormond Street Hospital NHS Foundation Trust, London, UK. Alain Combes, Sorbonne Université INSERM Unité Mixte de Recherche (UMRS) 1166, Institute of Cardiometabolism and Nutrition, Service de Médecine Intensive-Réanimation, Institut de Cardiologie, Assistance PubliqueHôpitaux de Paris (APHP) Hôpital Pitié-Salpêtrière, Paris, France. Michael Darmon, Unité de Médecine Intensive et Réanimation, Hôpital Universitaire Saint-Louis, Assistance Publique-Hôpitaux de Paris, and Faculté de Médecine,
Université Paris-Diderot, Sorbonne-Paris-Cité, Paris, France. Audrey De Jong, Saint Eloi ICU, Montpellier University Hospital, PhyMedExp, INSERM, CNRS, Montpellier, France. Sharon Einav, Surgical Intensive Care Unit, Shaare Zedek Medical Centre, and Hebrew University Faculty of Medicine, Jerusalem, Israel. Niall Ferguson, Interdepartmental Division of Critical Care Medicine, Departments of Medicine and Physiology, Institute of Health Policy, Management and Evaluation, University of Toronto and Department of Medicine, Division of Respirology, University Health Network and Sinai Health System. Morten Hylander Møller, Department of Intensive Care, Copenhagen University Hospital, Rigshospitalet, Copenhagen, Denmark and Centre for Research in Intensive Care (CRIC), Copenhagen University Hospital, Rigshospitalet, Copenhagen, Denmark. Ignacio Martin-Loeches, Department of Intensive Care Medicine, Multidisciplinary Intensive Care Research Organization (MICRO), St. James's Hospital, Dublin, Ireland. Paul Mayo, Division of Pulmonary, Critical Care and Sleep Medicine, Northwell Health LIJ/NSUH Medical Center, Zucker School of Medicine, Hofstra/Northwell, Hempstead, NY, USA. Geert Meyfroidt, Laboratory of Intensive Care Medicine, Academic Department of Cellular and Molecular Medicine, Katholieke Universiteit Leuven, Leuven, Belgium. Garyphallia Poulakou, 3rd Department of Internal Medicine, Sotiria General Hospital, Medical School, National and Kapodistrian University of Athens, Athens, Greece. Otavio Ranzani, Pulmonary Division, Heart Institute, Universidade de Sao Paulo, São Paulo, Brazil and Barcelona Institute for Global Health (ISGlobal), Barcelona, Spain. Claudio Sandroni, Istituto Anestesiologia e Rianimazione, Università Cattolica del Sacro Cuore, Fondazione Policlinico Universitario "Agostino Gemelli" IRCCS, Rome, Italy. Manu Shankar-Hari, Intensive Care National Audit \& Research Centre, London, United Kingdom and Department of Intensive Care, Guy's and St Thomas' NHS Foundation Trust, St Thomas' Hospital, London, United Kingdom and Division of Infection, Immunity and Inflammation, Kings College London, London, United Kingdom. 
Funding

Open Access funding enabled and organized by Projekt DEAL.

\section{Compliance with ethical standards}

\section{Conflicts of interest}

The authors declare no conflicts of interest.

\section{Open Access}

This article is licensed under a Creative Commons Attribution-NonCommercial 4.0 International License, which permits any non-commercial use, sharing, adaptation, distribution and reproduction in any medium or format, as long as you give appropriate credit to the original author(s) and the source, provide a link to the Creative Commons licence, and indicate if changes were made. The images or other third party material in this article are included in the article's Creative Commons licence, unless indicated otherwise in a credit line to the material. If material is not included in the article's Creative Commons licence and your intended use is not permitted by statutory regulation or exceeds the permitted use, you will need to obtain permission directly from the copyright holder. To view a copy of this licence, visit http://creativecommons.org/licen ses/by-nc/4.0/.

\section{Publisher's Note}

Springer Nature remains neutral with regard to jurisdictional claims in published maps and institutional affiliations.
Published online: 19 August 2020

\section{References}

1. Black N, van Rooyen S, Godlee F, Smith R, Evans S (1998) What makes a good reviewer and a good review for a general medical journal? JAMA 280:231-233

2. Allen TW (2013) Peer review guidance: how do you write a good review? J Am Osteopath Assoc 113:916-920

3. Tite L, Schroter S (2007) Why do peer reviewers decline to review? A survey. J Epidemiol Community Health 61:9-12

4. Neto MLR, Almeida HG, Esmeraldo JD, Nobre CB, Pinheiro WR, de Oliveira CRT, Sousa IDC, Lima OMML, Lima NNR, Moreira MM, Lima CKT, Júnior JG, da Silva CGL (2020) When health professionals look death in the eye: the mental health of professionals who deal daily with the 2019 coronavirus outbreak. Psychiatry Res 288:112972. https://doi.org/10.1016/j.psych res.2020.112972

5. Clarke M (2020) Mike Clarke: How can we avoid research waste during the covid-19 pandemic and plan for the future. BMJ Opin https://blogs .bmj.com/bmj/2020/04/21/mike-clarke-avoid-research-waste-covid -19-pandemic-plan-future/?utm_source =twitter\&utm_medium=socia I\&utm_term=hootsuite\&utm_content=sme\&utm_campaign=usage. 